\title{
S10 Table.
}

DLNR reported catch for 2009-2013 for reporting block 102, with catch value, and fraction of annual catch and annual value represented by each species.

\begin{tabular}{|c|c|c|c|c|c|c|c|}
\hline Year & Method & Species & $\begin{array}{r}\text { Lbs } \\
\text { caught }\end{array}$ & $\begin{array}{r}\mathrm{Kg} \\
\text { caught }\end{array}$ & Trophic Group & $\begin{array}{r}\% \text { of } \\
\text { annual } \\
\text { catch }\end{array}$ & $\begin{array}{r}\% \text { of } \\
\text { annual } \\
\text { value }\end{array}$ \\
\hline 2009 & Handline & Opelu & 4,744 & 2,152 & Planktivore & 50.7 & 44.1 \\
\hline 2009 & Handline & Uku & 2,065 & 937 & Apex Predator & 22.1 & 28.8 \\
\hline 2009 & Handline & Menpachi & 964 & 437 & Secondary consumer & 10.3 & 15.7 \\
\hline 2009 & Handline & Kahala & 545 & 247 & Apex Predator & 5.8 & $\overline{5.6}$ \\
\hline 2009 & Handline & Laenihi & 92 & 42 & Secondary consumer & 1.0 & 2.6 \\
\hline 2009 & Handline & Kamanu & 46 & 21 & Apex Predator & 0.5 & $\mathrm{~N} / \mathrm{A}$ \\
\hline 2009 & Handline & White ulua & 41 & 19 & Apex Predator & 0.4 & 0.6 \\
\hline 2009 & Handline & Taape & 9 & 4 & Secondary consumer & 0.1 & 0.1 \\
\hline 2009 & Net & Kona crab & 718 & 326 & Secondary consumer & 7.7 & $\mathrm{~N} / \mathrm{A}$ \\
\hline 2009 & Spear/Dive & Roi & 60 & 27 & Secondary consumer & 0.6 & 1.0 \\
\hline 2009 & Spear/Dive & Palani & 21 & 10 & Herbivore & 0.2 & 0.1 \\
\hline 2009 & Spear/Dive & Menpachi & 20 & 9 & Secondary consumer & 0.2 & 0.3 \\
\hline \multirow[t]{2}{*}{2009} & Troll & Kaku & 35 & 16 & Apex Predator & 0.4 & 1.1 \\
\hline & & TOTAL & 9,360 & 4,246 & & 100 & 100 \\
\hline 2010 & Handline & Opelu & 8,521 & 3,865 & Planktivore & 56.4 & 48.8 \\
\hline 2010 & Handline & Uku & 2,701 & 1,225 & Apex Predator & 17.9 & 23.2 \\
\hline 2010 & Handline & Kahala & 1,166 & 529 & Apex Predator & 7.7 & 7.3 \\
\hline 2010 & Handline & Laenihi & 598 & 271 & Secondary consumer & 4.0 & 10.2 \\
\hline 2010 & Handline & White ulua & 268 & 122 & Apex Predator & 1.8 & 2.4 \\
\hline 2010 & Handline & Taape & 162 & 73 & Secondary consumer & 1.1 & 0.6 \\
\hline 2010 & Handline & Akule & 160 & 73 & Planktivore & 1.1 & 1.1 \\
\hline 2010 & Handline & Opelu kala & 99 & 45 & Herbivore & 0.7 & 0.3 \\
\hline 2010 & Handline & Kamanu & 52 & 24 & Apex Predator & 0.3 & $\mathrm{~N} / \mathrm{A}$ \\
\hline 2010 & Handline & Weke nono & 49 & 22 & Secondary consumer & 0.3 & 0.4 \\
\hline 2010 & Handline & Aawa & 20 & 9 & Secondary consumer & 0.1 & 0.1 \\
\hline 2010 & Handline & Weke ula & 20 & 9 & Planktivore & 0.1 & 0.2 \\
\hline 2010 & Handline & Toau & 13 & 6 & Secondary consumer & 0.1 & 0.1 \\
\hline 2010 & Handline & Moana kale & 9 & 4 & Planktivore & 0.1 & 0.2 \\
\hline 2010 & Net & Kona crab & 721 & 327 & Secondary consumer & 4.8 & $\mathrm{~N} / \mathrm{A}$ \\
\hline 2010 & Spear/Dive & Day tako & 402 & 182 & Secondary consumer & 2.7 & 3.5 \\
\hline \multirow[t]{2}{*}{2010} & Spear/Dive & Menpachi & 160 & 73 & Secondary consumer & 1.1 & 1.6 \\
\hline & & TOTAL & 15,121 & 6,859 & & 100 & 100 \\
\hline 2011 & Handline & Uku & 6,524 & 2,959 & Apex Predator & 35.3 & 43.9 \\
\hline 2011 & Handline & Opelu & 1,772 & 804 & Planktivore & 9.6 & 7.9 \\
\hline 2011 & Handline & Menpachi & 591 & 268 & Secondary consumer & 3.2 & 4.6 \\
\hline 2011 & Handline & Akule & 565 & 256 & Planktivore & 3.1 & 2.9 \\
\hline 2011 & Handline & Kahala & 315 & 143 & Apex Predator & 1.7 & 1.6 \\
\hline 2011 & Handline & Laenihi & 230 & 104 & Secondary consumer & 1.2 & 3.1 \\
\hline 2011 & Handline & Taape & 45 & 20 & Secondary consumer & 0.2 & 0.1 \\
\hline 2011 & Handline & Opelu kala & 30 & 14 & Herbivore & 0.2 & 0.1 \\
\hline 2011 & Net & Opelu & 7,043 & 3,195 & Planktivore & 38.1 & 31.6 \\
\hline 2011 & Net & Kona crab & 768 & 348 & Secondary consumer & 4.2 & $\mathrm{~N} / \mathrm{A}$ \\
\hline \multirow[t]{2}{*}{2011} & Spear/Dive & Day tako & 591 & 268 & Secondary consumer & 3.2 & 4.1 \\
\hline & & TOTAL & 18,474 & 8,380 & & 100 & 100 \\
\hline
\end{tabular}




\begin{tabular}{|c|c|c|c|c|c|c|c|}
\hline 2012 & Handline & Uku & 1,997 & 906 & Apex Predator & 14.0 & 17.8 \\
\hline 2012 & Handline & Opelu & 1,265 & 574 & Planktivore & 8.9 & 7.5 \\
\hline 2012 & Handline & Laenihi & 386 & 175 & Secondary consumer & 2.7 & 6.9 \\
\hline 2012 & Handline & Akule & 327 & 148 & Planktivore & 2.3 & 2.3 \\
\hline 2012 & Handline & Kahala & 160 & 73 & Apex Predator & 1.1 & 1.0 \\
\hline 2012 & Handline & Weke nono & 125 & 57 & Secondary consumer & 0.9 & 1.0 \\
\hline 2012 & Handline & Menpachi & 110 & 50 & Secondary consumer & 0.8 & 1.1 \\
\hline 2012 & Handline & Weke ula & 39 & 18 & Secondary consumer & 0.3 & 0.3 \\
\hline 2012 & Handline & Kamanu & 28 & 13 & Apex Predator & 0.2 & $\mathrm{~N} / \mathrm{A}$ \\
\hline 2012 & Handline & Aweoweo & 26 & 12 & Planktivore & 0.2 & 0.2 \\
\hline 2012 & Handline & Taape & 18 & 8 & Secondary consumer & 0.1 & 0.1 \\
\hline 2012 & Net & Opelu & 7,610 & 3,452 & Planktivore & 53.5 & 45.2 \\
\hline 2012 & Net & Kona crab & 362 & 164 & Secondary consumer & 2.5 & $\mathrm{~N} / \mathrm{A}$ \\
\hline 2012 & Spear/Dive & Day tako & 1,373 & 623 & Secondary consumer & 9.7 & 12.6 \\
\hline 2012 & Spear/Dive & $\begin{array}{l}\text { Uhu parrot- } \\
\text { misc. }\end{array}$ & 248 & 112 & Herbivore & 1.7 & 2.2 \\
\hline 2012 & Spear/Dive & Menpachi & 70 & 32 & Secondary consumer & 0.5 & 0.7 \\
\hline 2012 & Spear/Dive & Kumu & 40 & 18 & Secondary consumer & 0.3 & 0.8 \\
\hline \multirow[t]{2}{*}{2012} & Spear/Dive & $\mathrm{Mu}$ & 31 & 14 & Secondary consumer & 0.2 & 0.3 \\
\hline & & TOTAL & 14,215 & 6,448 & & 100 & 100 \\
\hline 2013 & Handline & Opelu & 767 & 348 & Planktivore & 6.7 & 6.4 \\
\hline 2013 & Handline & Uku & 682 & 309 & Apex Predator & 6.0 & 8.5 \\
\hline 2013 & Handline & Kahala & 615 & 279 & Apex Predator & 5.4 & 5.6 \\
\hline 2013 & Handline & Akule & 499 & 226 & Planktivore & 4.4 & 4.8 \\
\hline 2013 & Handline & Laenihi & 107 & 49 & Secondary consumer & 0.9 & 2.7 \\
\hline 2013 & Handline & Opelu kala & 42 & 19 & Herbivore & 0.4 & 0.2 \\
\hline 2013 & Handline & Weke nono & 32 & 14 & Secondary consumer & 0.3 & 0.4 \\
\hline 2013 & Net & Opelu & 7,332 & 3,326 & Planktivore & 64.5 & 61.2 \\
\hline 2013 & Net & Kona crab & 493 & 224 & Secondary consumer & 4.3 & $\mathrm{~N} / \mathrm{A}$ \\
\hline 2013 & Spear/Dive & Day tako & 326 & 148 & Secondary consumer & 2.9 & 4.2 \\
\hline \multirow[t]{2}{*}{2013} & Troll & Uku & 479 & 217 & Apex Predator & 4.2 & 6.0 \\
\hline & & & 11,374 & 5,159 & & & \\
\hline
\end{tabular}

